\title{
APRENDIZAJE COLABORATIVO VS. APRENDIZAJE COOPERATIVO: UNA EXPERIENCIA DENTRO DEL CONTEXTO DE LA PROGRAMACIÓN DE COMPUTADORES EN INGENIERÍA DE SISTEMAS USANDO PROBLEM BASED LEARNING
}

\author{
Collaborative LeARning VS. COOPERATIVE LEARNing: AN EXPERIENCE \\ WITHIN THE CONTEXT OF THE COMPUTER PROGRAMIMING IN SYSTEMS \\ ENGINEERING USING PROBLEM-BASED LEARNING
}

Omar Iván Trejos Buriticá*

Recibido: 9 de junio de 2017

Aceptado: 4 de septiembre de 2017

\section{Resumen}

El presente artículo es uno de los productos de un proyecto de investigación en el aula, cuyo propósito fue comparar, desde lo experimental, dos enfoques de trabajo en grupo con el ánimo de tener elementos de juicio sólidos que permitieran inferir la conveniencia de uno de estos enfoques dentro del proceso de enseñanza y aprendizaje de la programación de computadores en un curso inicial de ingeniería de sistemas. Para ello se recurrió a un diseño experimental en el aula durante el desarrollo de la asignatura Programación I a lo largo de seis semestres, con la conformación de subgrupos de observación paralelos dentro del mismo curso. Se encontró que efectivamente la investigación en programas de ingeniería no solo es conveniente, sino muy necesaria para que los docentes puedan tener criterios firmes al momento de buscar caminos para que sus alumnos logren los objetivos de aprendizaje. Se concluye que si se aprovechan de la forma apropiada, los enfoques de aprendizaje colaborativo y de aprendizaje cooperativo son caminos que posibilitan la interacción, comunicación, superación, cohesión, autonomía y socialización; características altamente necesarias que el ingeniero de nuestros tiempos puede ir fomentando desde su proceso de formación como estudiante.

Palabras clave: aprendizaje colaborativo, aprendizaje cooperativo, ingeniería de sistemas, programación de computadores, trabajo en grupo.

\section{Abstract}

This article is one of the products of a research project in the classroom. The purpose was to compare, from the experimental, two approaches of group work with the aim of having solid elements of judgment. This would allow inferring the convenience of one of these methods, within the teaching and learning process of computer programming, in an initial course of systems engineering. For that purpose, an experimental design in the classroom during the development of the subject programming over six semesters was drawn, with the establishment of parallel observation subgroups within the same course. Indeed, the research in engineering programs is not only convenient

* Ph. D. en Ciencias de la Educación. Docente de planta, Universidad Tecnológica de Pereira, Pereira, Colombia. ORCID:http://orcid.org/0000-0002-3751-6014. omartrejos@utp.edu.co 
but very necessary for teachers to have consistent criteria at the time of searching ways for their students to achieve learning objectives. As a conclusion, if they take advantage of the appropriate form, the approaches to collaborative learning and cooperative learning are pathways that allow interaction, communication, improvement, cohesion, autonomy, and socialization; highly necessary characteristics that the engineer of our times can encourage their training process as a student.

Keywords: collaborative learning, cooperative learning, systems engineering, computer programming, group work

\section{INTRODUCCIÓN}

Una de las estrategias más utilizadas en los procesos de formación básica y profesional es la realización de actividades en grupos de trabajo. Con esta estrategia normalmente se busca la socialización de soluciones y la posibilidad de que los estudiantes conozcan otras opiniones frente a un problema común y se acostumbren a trabajar con personas que discrepan o coinciden con ellos, así como fortalecer el debate, la diferencia y las coincidencias como caminos de interacción y fortalecimiento académico.

Este artículo se deriva de un proyecto de investigación en el que se pretende encontrar caminos que posibiliten aprovechar las cualidades, habilidades y competencias que los estudiantes universitarios usan en pro de ellos mismos y de los demás, de manera que cada uno se nutra de los otros y puedan trabajar en conjunto dentro de visiones diferentes, pero con objetivos comunes. Normalmente, en los procesos de formación de ingenieros, los docentes acuden a la estrategia del trabajo en grupo sin mayor conocimiento que la organización aleatoria y la designación de una tarea común son otros modelos de trabajo en grupo. Algunas veces se escoge a alguien del grupo para sustentar el resultado y en otras oportunidades se hacen pruebas escritas para validar lo que el grupo presenta, como una forma de verificar el nivel de aprendizaje individual de cada uno de los integrantes.

Esta forma de incorporar el trabajo en grupo en los salones de clases ha sido la tradicional; pero la pregunta que subyace es: ¿cómo hacer para que cada estudiante fortalezca sus habilidades y competencias, su nivel y su ritmo de aprendizaje, a partir de la interacción con otros estudiantes con los que realice actividades académicas en el formato de grupos de trabajo? Este artículo resume los resultados de una experiencia investigativa en el aula con sentido cuantitativo y cualitativo a través de la cual se ha querido encontrar una respuesta que dé elementos de juicio a los ingenieros docentes al momento de incorporar la estrategia del trabajo en grupo en sus asignaturas, especialmente en las que se refiere a la línea de programación de computadores dentro de ingeniería de sistemas.

La necesidad de encontrar respuestas a esta pregunta y de encontrar caminos que capitalicen las bondades del trabajo en grupo es lo que ha llevado a que se plantee esta investigación, cuyos resultados se exponen en este artículo. Lo innovador de este documento radica en que pocas veces se realizan investigaciones en el aula con grupos paralelos en asignaturas de programas de ingeniería, dado que normalmente muy pocas veces la docencia en ingeniería se nutre de las teorías, modelos, estrategias, enfoques y prácticas de las ciencias de la educación.

Desde la perspectiva de la ingeniería de sistemas, este estudio se justifica porque es muy importante que hoy en día los egresados tengan capacidad de socialización y articulación con otros profesionales, amén de que hoy no se concibe esta ingeniería sin la transdisciplinariedad y la interdisciplinariedad, que la hacen fuerte en su relación con otras áreas y núcleos de conocimiento. Para el planteamiento de esta investigación se acudió a literatura especializada tanto en teorías de aprendizaje, como en la temática disciplinar que está relacionada con la programación de computadores, además de la experiencia del autor y de otras experiencias de autores que han pu- 
blicado al respecto y de investigadores que han interactuado por diferentes medios con el autor de este artículo.

El presente estudio es un producto del proyecto de investigación 6-16-13, titulado Desarrollo de un Modelo Metodológico para el Aprendizaje de la Programación en Ingeniería de Sistemas Basado en Aprendizaje Significativo, Aprendizaje por Descubrimiento y el Modelo 4Qde Preferencias de Pensamiento. ${ }^{1}$ La temática que se propone como objeto investigativo tiene una particular relevancia por el posible impacto que puede causar en ingenieros docentes y en todos aquellos docentes de programas de formación tecnológica. Este estudio se desarrolló con los estudiantes del curso Programación I de Ingeniería de Sistemas y Computación de la Facultad de Ingenierías de la Universidad Tecnológica de Pereira, durante los semestres I y II de 2014, 2015 y 2016. El estudio se realizó bajo los parámetros y metodologías asociadas con la investigación cuantitativa y cualitativa en educación y se acudió a las teorías de aprendizaje colaborativo, aprendizaje cooperativo, aprendizaje basado en problemas, active learning y un modelo de clasificación de estudiantes basado en la expereincia docente del autor.

Este artículo se organiza bajo el estándar IMRYD. El IMRYD inicia con una introducción complementada por un marco teórico. Después presenta la metodología que se utilizó en la investigación y luego muestra los resultados obtenidos para plantear unas reflexiones, a manera de discusión, a partir de ellos. Al final se presentan unas conclusiones, que se espera enriquezcan los debates al respecto de la formación en programación en ingeniería de sistemas y otras ingenierías que se hagan en las salas de profesores respectivas en referencia con la incorporación de la estrategia de trabajo en grupo.

A manera de hipótesis se plantean dos preguntas: 1) ¿es posible fortalecer y mejorar significativamente el proceso de aprendizaje de la programación de computadores por parte de los estudiantes (de manera individual) si se adoptan estrategias como aprendizaje colaborativo y aprendizaje cooperativo a partir del planteamiento de problemas? y 2) ¿es posible mirar comparativamente las dos estrategias enunciadas y poder establecer criterios que determinen la más apropiada en el campo del aprendizaje de la programación de computadores? La respuesta a estas preguntas es el contenido del presente artículo.

\section{MARCO TEÓRICO}

El aprendizaje basado en problemas (APB o PBL, por sus siglas en inglés) es un enfoque pedagógico centrado en el estudiante en el cual los alumnos aprenden acerca de un tema a través de la experiencia de resolver problemas abiertos, para los cuales se entrega un determinado material (Barrell, 2006). El proceso de aprendizaje basado en problemas no se enfoca en la resolución de un problema a partir de una única solución, sino que posibilita el desarrollo de habilidades y atributos que son deseables en los estudiantes. Estos incluyen adquisición de conocimiento, comunicación y colaboración en el interior de un grupo e interacción entre los alumnos.

La incorporación del APB involucra trabajo en pequeños grupos de aprendices. Cada estudiante asume un determinado rol dentro del grupo, que puede ser formal o informal y que se rota entre todos (Duch, Groh y Allen, 2001). Esta estrategia se enfoca en la relación entre estudiantes y los razonamientos que son necesarios para construir su propio aprendizaje. En términos generales, APB incluye clarificación de términos, definición de problemas, lluvia de ideas, estructuración de hipótesis, definición de objetivos de aprendizaje, estudio independiente y síntesis. Lo que se busca es que los estudiantes definan claramente lo que saben, lo que necesitan y cómo y dónde acceder a nueva información que puede conducir a la resolución de un problema.

\footnotetext{
${ }_{1}^{1}$ Proyecto avalado por el Consejo de Facultad de Ingenierías y aprobado por la Vicerrectoría de Investigaciones, Extensión e Innovación, de la Universidad Tecnológica de Pereira.
} 
E1 rol del tutor es facilitar el aprendizaje y brindar soporte, guiarlo y monitorear todo el proceso. El tutor debe construir una relación cercana con los estudiantes y animarlos a resolver el problema planteado, mientras ellos mejoran su proceso de aprendizaje. Este proceso se basa en constructivismo. APB representa un paradigma que alterna con la filosofía tradicional de la enseñanza y el aprendizaje que normalmente se basa en clases magistrales. Los requerimientos para organizar una sesión APB difieren de la clase tradicional y normalmente necesitan más tiempo de preparación, más recursos y mayor soporte en el aprendizaje de los pequeños grupos. Los principios de un proceso APB se basan en el acompañamiento del aprendiz, la guía en los logros que el mismo defina, la independencia del estudiante, la autorregulación, el aprendizaje a partir de pequeños grupos, la discusión en el interior de los grupos, el material de apoyo y el fomento de la comunicación, la interacción, la discusión y el debate de los integrantes de dichos grupos (Paz, 2009).

El aprendizaje colaborativo puede definirse como una sombrilla bajo la cual se agrupan diferentes enfoques en educación, que implica un esfuerzo intelectual conjunto por parte de los estudiantes y la colaboración y acompañamiento de sus profesores. El aprendizaje colaborativo hace referencia a metodologías y entornos en donde los estudiantes se involucran en una tarea común en la que cada uno depende de la colaboración (Collazos, Guerrero, Pino y Ochoa, 2002). Igualmente, involucra la organización de pequeños grupos, de forma que cada estudiante pueda maximizar su propio aprendizaje y el de sus pares.

Es un proceso de creación compartida en el cual dos o más estudiantes interactúan para crear un proceso colaborativo que posibilita aprender un determinado concepto de un área o una disciplina. El aprendizaje colaborativo puede incluir escritos colaborativos, proyectos en grupo y otras actividades conjuntas en las que la participación activa de cada alumno es determinante. El término se refiere a un método de instrucción en el que los estudiantes, a partir de sus diferentes niveles de desempeño, trabajan en pequeños grupos buscando un objetivo común. Los cinco elementos que se involucran en el aprendizaje colaborativo son: interdependencia positiva, responsabilidad individual y grupal, habilidades interpersonales en pequeños grupos, interacción frente a frente y procesamiento en grupo (Dooly, 2008).

Es de anotar que la interacción frente a frente ha tomado un giro muy interesante en la actualidad, pues los nuevos canales de comunicación posibilitan una interacción de este tipo sin que involucre la presencialidad directa de los participantes (Small, 2011). El éxito del aprendizaje colaborativo en el aula implica: definición clara de los objetivos del grupo, estructuración de grupos pequeños, definición de normas claras y flexibles para el grupo, promoción de la comunicación entre los integrantes, asignación de roles, establecimiento de mecanismos de evaluación, adopción del proceso de aprendizaje como parte de la evaluación, uso de diferentes estrategias de cohesión y aprendizaje en grupo y acompañamiento permanente por parte del docente.

Del aprendizaje cooperativo puede decirse que sus objetivos están estructurados para promover los esfuerzos de cooperación, competitividad y presencia individual (Medina, 2012). En todo salón de clases, las actividades instruccionales son direccionados hacia el logro de unos objetivos definidos y todo conduce hacia ellos. Un objetivo de aprendizaje es un estado futuro deseado de demostración de una competencia en el tema de un área específica (Gómez, Gómez y González, 2007). El objetivo especifica los caminos por los cuales los estudiantes podrán interactuar con otros y con el profesor durante una sesión de acompañamiento.

En el salón de clases ideal, todos los estudiantes deberían aprender cómo trabajar cooperativamente con otros, cómo competir divirtiéndose y cómo trabajar de manera autónoma en sus propios objetivos (Ausubel, 1986). El docente decide cuál es el objetivo que debe alcanzarse en cada sesión. La estructura de objetivos más importante y la que debe usarse la mayor parte del tiempo en situaciones de aprendizaje es la cooperación. La cooperación trabaja conjuntamente 
para alcanzar objetivos comunes. Con situaciones cooperativas, cada estudiante busca sus objetivos individuales beneficiándose de los demás y, al final, se beneficia todo el grupo (Mabrouk, 2007). El aprendizaje cooperativo es una estrategia instruccional de pequeños grupos en la que los estudiantes pueden trabajar para maximizar su propio aprendizaje y, con ello, el aprendizaje de los demás.

Se contrasta de manera muy clara con lo puramente competitivo (los estudiantes trabajan en contra de otros para alcanzar sus objetivos académicos más altos que pocos pueden obtener) e individual (los estudiantes trabajan solos sin relacionarse con los demás). En el aprendizaje cooperativo se evalúan los esfuerzos de cada estudiante sobre la base de unos criterios grupales, mientras que en el aprendizaje competitivo los criterios son individuales. Es claro que en ciertos momentos se requieran algunas valoraciones competitivas; pero lo cooperativo pareciera ser más apropiado, en la mayoría de las situaciones, para que el nivel de aprendizaje sea común a todos los alumnos (Gillies, 2007).

Puede decirse que los beneficios de trabajar en grupo son bastante obvios si se trata de propósitos de aprendizaje. El aprendizaje cooperativo es una estrategia instruccional que direcciona simultáneamente las habilidades académicas y las sociales en los estudiantes. Es una estrategia instruccional y se ha detectado como muy exitosa en el salón de clases porque incrementa el nivel de interdependencia positiva entre los estudiantes, dotándolos con las herramientas necesarias para aprender de otros. Los estudiantes trabajan en la búsqueda plena de sus objetivos que se han definido claramente. Es un enfoque de grupo en donde el éxito del conjunto depende de que cada uno ponga lo mejor de sí.

Por su parte, el aprendizaje colaborativo es bastante útil cuando los grupos de estudiantes trabajan juntos para entender el significado de conceptos o para resolver o crear artefactos o productos que se derivan de su proceso de aprendizaje. El aprendizaje colaborativo redefine la relación entre el docente y el estudiante en el salón de clases, porque las actividades pueden incluir escritura colaborativa, proyectos en grupo, resolución conjunta de problemas, debates, grupos de estudio y otras actividades en las que los estudiantes trabajan en grupo para explorar la respuesta a una pregunta o para la creación de un proyecto significativo.

Puede pensarse en el active learning como un enfoque instruccional en el que los estudiantes se conectan con el material que estudian a través de la lectura, escritura, comentarios y reflexiones al respecto (Bonwell y Eison, 1991). El active learning es diferente a los modos tradicionales de instrucción en los que los profesores son los que más hablan y los estudiantes son pasivos. Los estudiantes y sus necesidades de aprendizaje son el centro del active learning. Hay muchas estrategias de enseñanza que pueden ser utilizadas para articular a los estudiantes de manera activa en el proceso de aprendizaje, como discusiones en grupo, resolución de problemas, estudios de casos, juego de roles, ejercicios de escritura y grupos estructurados de aprendizaje. Los beneficios de incorporar estas actividades son muchos, e incluyen: mejoramiento sustancial en las habilidades de pensamiento crítico, incremento en la retención y transferencia de nueva información, incremento en la motivación y mejora notoria en las habilidades interpersonales.

La utilización del active learning no implica abandonar el formato de la clase magistral, sino que permite ocupar mayor tiempo en clase. Los docentes que usan active learning frecuentemente durante el periodo (cada 15 minutos o algo así) les dan a los estudiantes unos minutos para trabajar con la información que le están proporcionando. Ellos pueden preguntar a los estudiantes para que respondan determinadas preguntas, resumir conceptos importantes en escritura o comparar sus notas con un compañero. Para algunas clases magistrales, el uso del aprendizaje activo puede ser un poco retador, dependiendo del tamaño del curso y de las limitaciones del salón de clases tales como las sillas fijadas al suelo (Prince, 2004). Dividir los estudiantes en grupos en estas circunstancias no es posible; pero otras estrategias como la escritura individual o el trabajo por pares son viables y podrían generar muy buenos resultados. 
El aprendizaje activo es, pues, un enfoque centrado en el estudiante; aquí, la responsabilidad del aprendizaje está situada más en el alumno, que normalmente deberá trabajar en colaboración con otros compañeros. En el active learning los docentes son facilitadores, en comparación con su papel tradicional de ser proveedores de información. La presentación de hechos, que normalmente se introducen a través de las clases magistrales, es poco útil en comparación con las discusiones en clase, la resolución de problemas, el aprendizaje cooperativo y los ejercicios de escritura (Hall, Waitz, Brodeur, Soderholm y Nasr, 2002). Otros ejemplos de técnicas del active learning son el juego de roles, los estudios de caso, los proyectos en grupo, el trabajo por parejas, la enseñanza por parejas, los debates, el aprendizaje in situ y las demostraciones cortas seguidas por discusiones en clase.

La clasificación de estudiantes se ha basado en la experiencia tanto del autor del presente artículo, como de otros docentes y/o investigadores. Según las conversaciones sostenidas, los estudiantes podrían ubicarse en tres categorías: los estudiantes altamente talentosos (AT), los medianamente talentosos (MT) y los bajamente talentosos (BT).

Podría definirse a los estudiantes AT como aquellos que ya tienen un conocimiento disciplinar bastante sólido, que sus habilidades y competencias están conectadas con dicho conocimiento disciplinar y que aprovechan cualquier oportunidad para aplicarlo. Los estudiantes BT son aquellos que cumplen con una de estas tres condiciones: a) no tienen un conocimiento disciplinar en ningún nivel, b) sus habilidades y competencias no están en correspondencia con los requerimientos de la asignatura y/o del programa de formación profesional y c) no manifiestan mayor interés de involucrarse en situaciones en donde dicho conocimiento pudiera se aplicado.

Los estudiantes MT son los que tienen un conocimiento disciplinar débil y muy difuso; posiblemente tienen habilidades y competencias que son afines con los requerimientos del programa de formación, pero esta habilidades aún no ha aflorado y, por lo tanto, muchas veces ni siquiera ellos lo saben y participan tímidamente en las situaciones en las que el conocimiento requerido debe aplicarse (aunque muestran gran interés en estar allí).

La enseñanza de la programación de computadores se ha convertido en un reto que tiene tres aristas. De una parte, el reto institucional de lograr que se den todas las condiciones académico administrativas para que los alumnos efectivamente aprendan a programar; de otra, el reto de los docentes de alcanzar este objetivo en la mayoría de estudiantes (ojalá fuera en todos), de manera que al finalizar un curso de programación todos hayan alcanzado sus objetivos de aprendizaje, y, por último, el reto de los estudiantes de aprovechar los diferentes estilos de enseñanza para alcanzar el objetivo de aprender a programar, combinando lo que reciben con sus propias habilidades con la disciplina de estudio dentro del marco sistemático de una asignatura (Trejos, 2013).

\section{Metodología}

El presente proyecto de investigación fue realizado en el curso Programación I de Ingeniería de Sistemas y Computación de la Facultad de Ingenierías, de la Universidad Tecnológica de Pereira, durante los semestres I y II de 2014, 2015 y 2016. Con el ánimo de obtener resultados y poder compararlos posteriormente, cada grupo se dividió en dos subgrupos: uno se destinó para trabajar bajo el enfoque de aprendizaje colaborativo, y el otro, para trabajar bajo el enfoque de aprendizaje cooperativo.

Los grupos que trabajaron con aprendizaje colaborativo tuvieron tareas, talleres y evaluaciones escritas designadas por el docente y con responsabilidad primaria del lider -escogido por los mismos integrantes del grupo y que normalmente era un estudiante tipo AT-, quien se encargaba de realizar la socialización y comprensión de las actividades propuestas y planteaba posibles soluciones (a manera de explicación), de forma que los estudiantes pudieran participar dependiendo del nivel de aprendizaje que fueran experimentando y a su propio ritmo. E1 docente siempre estuvo atento a la evolución de las actividades. Pocas veces de desviaron de su objetivo central. 
En los grupos que trabajaron con aprendizaje cooperativo recibieron las mismas tareas, talleres y evaluaciones. En estos grupos no hubo un líder como tal, sino que el docente, consciente del perfil de cada estudiante, asignó un labor específica a cada estudiante dentro de la actividad a desarrollar. La socialización y comprensión de las actividades se hizo entre todos los integrantes del grupo y la asesoría del docente siempre estuvo a la orden del día. La tabla 1 muestra el resumen de los cursos y la cantidad de estudiantes involucrados en el proceso.

Tabla 1. Estudiantes participantes en la investigación

\begin{tabular}{ccccc}
\hline \multirow{2}{*}{ Año } & Sem & $\begin{array}{c}\text { Ap } \\
\text { Colab }\end{array}$ & $\begin{array}{c}\text { Ap } \\
\text { Coop }\end{array}$ & Tot \\
\hline \multirow{2}{*}{2014} & I & 10 & 9 & 19 \\
& II & 10 & 11 & 21 \\
\multirow{2}{*}{2015} & I & 10 & 10 & 20 \\
& II & 11 & 11 & 22 \\
2016 & I & 11 & 10 & 21 \\
& II & 11 & 11 & 22 \\
\hline Total ests. & & 63 & 62 & 125 \\
\hline
\end{tabular}

Dado que para el desarrollo de este proyecto de investigación se adoptó un modelo de clasificación basado en la experiencia (que ya se explicó en el apartado anterior), a partir de la observación directa, interacción con los estudiantes y pruebas escritas, las primeras dos semanas se destinaron para ubicar a cada estudiante en su respectiva categoría. La tabla $2 \mathrm{~A}$ presenta la distribución de los estudiantes en cada categoría y la tabla 2B presenta la conformación de los grupos según la clasificación adoptada.

Tabla 2A. Distribución de estudiantes

\begin{tabular}{lccccc}
\hline Año & Sem. & AT & MT & BT & Total \\
\hline \multirow{2}{*}{2014} & I & 4 & 11 & 4 & 19 \\
& II & 5 & 12 & 4 & 21 \\
2015 & I & 5 & 10 & 5 & 20 \\
& II & 6 & 11 & 5 & 22 \\
2016 & I & 5 & 10 & 6 & 21 \\
& II & 6 & 10 & 6 & 22 \\
\hline Total & & 31 & 64 & 30 & 125 \\
\hline
\end{tabular}

Tabla 2B. Conformación de grupos de trabajo

\begin{tabular}{ccccc}
\hline Año & Sem & $\begin{array}{c}\text { Grps } \\
\text { ApCol }\end{array}$ & $\begin{array}{c}\text { Grps } \\
\text { ApCoop }\end{array}$ & Total \\
\hline \multirow{2}{*}{2014} & I & 2 & 2 & 4 \\
& II & 3 & 2 & 5 \\
2015 & 1 & 3 & 2 & 5 \\
& II & 3 & 3 & 6 \\
2016 & I & 3 & 2 & 5 \\
& II & 3 & 3 & 6 \\
\hline
\end{tabular}


En la distribución de los estudiantes se procuró que cada grupo contara, en lo posible, con al menos un estudiante de cada categoría. Por razones apenas naturales, hay que entender que, dada la información de la tabla $2 \mathrm{~A}$, en algunos grupos se tuvo que ubicar a varios estudiantes de una misma categoría. Para el desarrollo de la asignatura Programación I, se proyectaron tres talleres y tres pruebas escritas, cada uno con un valor de $10 \%$, para un valor total (por periodo temático) de $20 \%$. De la misma manera se programó una evaluación final con un valor de $40 \%$, para completar el $100 \%$ de la nota definitiva. Como factor motivacional se le informó a los estudiantes que la nota final tendría un incremento de 1 unidad.

Es de anotar que, aprovechando la clasificación de estudiantes que se ha adoptado, se pretendió con este proyecto de investigación resaltar el posible carácter de liderazgo de los estudiantes AT, así como intentar encauzar al estudiante BT en el el perfil del programa Ingeniería de Sistemas. También se quiso capitalizar el talento oculto de los estudiantes MT, de forma que pudiera aflorar en ellos esas capacidades que les permitirán alcanzar fácilmente sus objetivos de aprendizaje.

\section{Resultados}

La tabla 3 el resultado de las notas definitivaas obtenidas por cada grupo según la clasificación adoptada en su confomación y su distribución.

Tabla 3. Notas obtenidas por los grupos (Grp)

\begin{tabular}{|c|c|c|c|c|c|c|c|c|c|c|}
\hline Año & Sem & $\begin{array}{c}\text { Grp. } \\
1\end{array}$ & $\begin{array}{c}\text { Grp } \\
2\end{array}$ & $\begin{array}{c}\text { Grp } \\
3\end{array}$ & $\begin{array}{c}\text { Grp } \\
4\end{array}$ & $\begin{array}{c}\text { Grp } \\
5\end{array}$ & $\begin{array}{c}\text { Grp } \\
6\end{array}$ & $\begin{array}{l}\text { Grps } \\
\text { ApCol }\end{array}$ & $\begin{array}{c}\text { Grps } \\
\text { ApCoop }\end{array}$ & $\begin{array}{c}\text { Total } \\
\text { Grp }\end{array}$ \\
\hline \multirow{2}{*}{2014} & 1 & 3.8 & 3.7 & 3.7 & 3.6 & $x$ & $x$ & 3.75 & 3.65 & 4 \\
\hline & II & 3.5 & 3.6 & 3.8 & 3.8 & 3.9 & $x$ & 3.63 & 3.85 & 5 \\
\hline \multirow{2}{*}{2015} & 1 & 3.3 & 3.4 & 3.7 & 3.5 & 3.4 & $x$ & 3.46 & 3.45 & 5 \\
\hline & II & 3.7 & 3.7 & 3.6 & 3.5 & 3.5 & 3.4 & 3.66 & 3.5 & 6 \\
\hline \multirow{2}{*}{2016} & I & 3.8 & 3.8 & 4.1 & 3.6 & 3.8 & $x$ & 4.00 & 3.70 & 5 \\
\hline & II & 3.9 & 3.9 & 4.0 & 4.1 & 4.2 & 4.1 & 3.93 & 4.13 & 6 \\
\hline
\end{tabular}

Las celdas sombreadas corresponde a los grupos que trabajaron con aprendizaje colaborativo, y las celdas no sombreadas, a los grupos que trabajaron con aprendizaje cooperativo. Al finalizar el grupo se abrió un espacio escrito de opinión para que los estudiantes de manera anónima, identificando solamente el enfoque del grupo en el que trabajaron durante todo el semestre, destacaran las ventajas y desventajas de lo realizado y expusieran libremente si consideraban que habían aprendido a programar.

La tabla 4 presenta los elementos más destacados de dichas respuestas. La columnas ApCol y ApCoop representan la cantidad de estudiantes que respondieron en consonancia con la descripción que aparece en la columna Opinión.

Los ítems denotados como ventajas y desventajas se recolectaron a lo largo del semestre a partir de la opinión verbal de los mismos estudiantes. El propósito con cada uno de los perfiles de los estudiantes, además de posibilitar el aprendizaje de la teoría y la práctica que subyace a un primer curso de programación en lo que se refiere a sus conceptos básicos, fue explicado en el último párrafo del apartado donde se expone la metodología. Se informó este propósito desde el principio a los estudiantes y al finalizar el curso se les solicitó que explicaran por escrito (de manera anónima e indicando el grupo en el cual había participado) si dichos propósitos se habían alcanzado. La tabulación de las respuestas obtenidas se presentan en la tabla 5. 
Tabla 4. Opinión de los estudiantes

\begin{tabular}{lcc}
\hline \multicolumn{1}{c}{ Opinión } & ApColab & ApCoop \\
\hline Ventaja & 35 & 22 \\
\hline Mayor interacción con compañeros & 46 & 13 \\
Intercambio de opiniones & 56 & 52 \\
Se aprende más & 36 & 55 \\
Se asumen responsabilidades & 19 & 31 \\
Se ocupa uno de algo específico & 49 & 23 \\
Hay mayor camaradería & 42 & 11 \\
Mayor comunicación & & \\
\hline Desventaja & 11 & 29 \\
\hline Poca interacción & 21 & 36 \\
Poco intercambio de opiniones & 10 & 12 \\
Se aprende menos & 17 & 44 \\
No se asumen responsabilidades & 12 & 39 \\
Uno se ocupa de varias cosas & 11 & 45 \\
Hay poca camaradería & 8 & 49 \\
Hay poca comunicación & & \\
\hline
\end{tabular}

Tabla 5. Opinión final de los estudiantes

\begin{tabular}{|c|c|c|c|c|c|c|c|c|}
\hline \multirow{2}{*}{ Pregunta } & \multirow{2}{*}{ Grp } & \multicolumn{2}{|c|}{ AT } & \multicolumn{2}{|c|}{ MT } & \multicolumn{2}{|c|}{ BT } & \multirow{2}{*}{ Tol } \\
\hline & & Sí & No & sí & No & Sí & No & \\
\hline \multirow{2}{*}{$\begin{array}{l}\text { ¿Le sirvió la metodología para } \\
\text { aprender a programar? }\end{array}$} & $\begin{array}{l}\text { Ap } \\
\text { Col }\end{array}$ & 29 & 0 & 31 & 9 & 16 & 4 & 63 \\
\hline & $\begin{array}{l}\text { Ap } \\
\text { Coop }\end{array}$ & 2 & 0 & 14 & 10 & 7 & 3 & 62 \\
\hline Total & & 31 & & 64 & & 30 & & 125 \\
\hline \multirow{2}{*}{$\begin{array}{l}\text { ¿Se logró el propósito planteado } \\
\text { dentro de su perfil de clasificación? }\end{array}$} & $\begin{array}{l}\text { Ap } \\
\text { Colab }\end{array}$ & 30 & 0 & 40 & 13 & 18 & 6 & 63 \\
\hline & $\begin{array}{l}\text { Ap } \\
\text { Coop }\end{array}$ & 1 & 0 & 8 & 3 & 4 & 2 & 62 \\
\hline Total & & 31 & & 64 & & 30 & & 125 \\
\hline
\end{tabular}

\section{Discusión}

Los resultados obtenidos en el desarrollo del proyecto de investigación permiten hacer algunas reflexiones al respecto a manera de discusión. En primer lugar debe admitirse que si bien un periodo de seis semestres permite realizar un buen análisis en estos datos, no cabe duda de que las inferencias pueden ser mucho más sólidas si se amplía el tiempo de análisis y se realiza este mismo proyecto de investigación en otras asignaturas y otras carreras. Una muestra de 125 estudiantes en total permite que se tenga un panorama importante en relación con los resultados obtenidos.

En referencia con el modelo de clasificación de los estudiantes, debe decirse que este es producto de la experiencia investigativa, no solo del autor del presente artículo, sino también de otros docentes e investigadores. En la medida en que es un modelo que se basa en una experiencia que no es menospreciable, el proceso de ubicación de los estudiantes en su respectiva categoría podría mejorar. Es de anotar que en este proyecto se fortaleció la observación, el uso de pruebas escritas 
y el diálogo permanente con los alumnos durante las dos primeras semanas, con el ánimo de tener una percepción de la categoría de cada estudiante. Es posible que este proceso de clasificación de los estudiantes no tenga una certeza del $100 \%$ (como sucede con procesos de clasificación más elaborados); pero se puede afirmar que el proceso tiene una confiabilidad suficiente como para que los resultados permitan realizar inferencias igualmente confiables. Debe anotarse que un proceso de clasificación de estas características tiene que ser muy ágil dado que en este tipo de investigaciones el tiempo cuenta mucho, pues un semestre solamente dura dieciséis semanas.

La conformación de grupos de trabajo se diseñó para que cada grupo tuviera al menos un estudiante de cada categoría. Dado que la franja mayoritaria de estudiantes corresponde a los que se pueden clasificar como medianamente talentosos (MT), se procedió a conformar los grupos de trabajo con la participación de varios de ellos en cada grupo. Por su parte, los estudiantes altamente talentosos (AT) y bajamente talentosos (BT) fueron menores en cantidad; pero suficientes para poder lograr que cada grupo contara con al menos uno de ellos. La idea de hacerlo así es que se pudiera complementar las habilidades y talentos de cada estudiante con los de las otras categorías. Como parte propia del diseño de esta investigación, se supuso que si se conforma un grupo con estudiantes de cada categoría, la complementación, colaboración y cooperación podría ser mayor. Esta es una premisa que se planteó a partir de teorías administrativas de conformación de equipos eficientes de trabajo.

En referencia con las notas obtenidas con los grupos (ver tabla 3), se puede notar que no son mayores las diferencias entre las notas cuantitativas de los grupos de aprendizaje colaborativo y las de los grupos de aprendizaje cooperativo; sin embargo, se constató una diferencia levemente favorable a los grupos bajo aprendizaje colaborativo, pero se deja en claro que la tendencia no es altamente significativa. Las notas no son altas, pero se mantienen dentro de un rango entre 3.5 (como nota mínima) y 4.3 (como nota máxima). Si bien es claro que las notas no siempre reflejan el nivel de conocimiento adquirido de los estudiantes, tampoco se puede desconocer que la rigurosidad del proceso permite hacer algunas inferencias confiables.

La opinión de los estudiantes evidencia una clara comodidad de ellos, que tiende más hacia el trabajo colaborativo que al trabajo cooperativo. Si bien en lo puramente cuantitativo las notas no muestran mayor diferencia (aunque sí evidencian una tendencia notoria), la complementación del análisis cuantitativo con la opinión cualitativa de los estudiantes permite proponer algunas conclusiones. Según la tabla 4, los estudiantes parecieran encontrar muchas más ventajas en la participación de grupos bajo aprendizaje colaborativo que bajo los parámetros que se adoptaron bajo el aprendizaje cooperativo.

Se destacan resultados como la importancia que le dan los estudiantes al intercambio de opiniones y la mayor comunicación que posibilita el tabajo colaborativo. Por su parte, la designación de responsabilidades individuales es un factor que destacan como ventajas supremas en los grupos bajo aprendizaje cooperativo. Llama la atención que los resultados de un ítem "cómo se aprende más" o "cómo se aprende menos" sea tan equilibrado según la opinión de los participantes del proceso. Según eso, parece que tienen la impresión de que aprenden lo mismo en cualquiera de los dos enfoques investigativos.

Es por esta razón que se optó por recoger opiniones que de una u otra forma resumían la experiencia de los estudiantes en relación con las dos preguntas clave que se presentan en la tabla 5 . Según estas opiniones, las ventajas del enfoque de aprendizaje colaborativo son notoriamente superiores a las opiniones favorables del enfoque proporcionado por el aprendizaje coopeativo y, en síntesis, parece que a los estudiantes les gustó mucho más como enfoque de aprendizaje los elementos de juicio que da el aprendizaje colaborativo. Es de anotar que la cantidad de estudiantes no coincide exactamente con la cantidad original, debido a que algunos estudiantes se retiraron por razones de índole personal y de preferencias, mas no por razones del impacto académico sobre ellos. 
Vale la pena resaltar que en el desarrollo de este tipo de estudios se puede acudir a algunos elementos que pueden fortalecer las conclusiones y perfilar de manera más detallada los resultados obtenidos a partir del análisis de variables como el género y la edad. Dado que todos los estudiantes eran primíparos y que ninguno superaba los 22 años, se optó por considerarlo como un grupo homogéneo para este estudio. Factores de índole social, cultural y económico podrán ser útiles para analizar en posteriores estudios.

De la misma manera, en otro artículo de estas características se pueden detallar actividades internas del trabajo en grupo, bien, a la luz del aprendizaje cooperativo, o bien, a la luz del aprendizaje colaborativo, tal como lo sugieren algunos investigadores. En este artículo se omitieron los análisis detallados de la dinámica propia de cada grupo, pues se quisieron presentar los resultados generales allende la filigrana analítica investigativa que se puede derivar de este tipo de investigaciones.

\section{Conclusiones}

El desarrollo de investigaciones en el aula dentro de procesos formativos de ingenieros de sistemas no son muy comunes, no solo porque desde la ingeniería solo hasta ahora se está empezando a mirar la relación enseñanza y aprendizaje desde una optica diferente a la tradicional, sino porque los docentes ingenieros, en su amplia mayoría, carecen del conocimiento acerca de los fundamentos, las teorías y los modelos que proveen las ciencias de la educación.

Los resultados obtenidos en esta investigación indican que tanto el aprendizaje colaborativo como el aprendizaje cooperativo tienen ventajas dentro de un proceso de formación de ingenieros; sin embargo, todo parece indicar que en los primeros semestres es más útil la incorporación de técnicas de aprendizaje colaborativo dentro de las actividades grupales que se desarrollan en el aula. Por su parte, en semestres avanzados (léase semestres superiores al quinto semestre), la utilización de estrategias propias del aprendizaje cooperativo podría ser mas efectiva dada la formación que hasta ese momento le permite al estudiante desempeñarse dentro de unos roles determinados.

La cohesión que se genera en el interior de los grupos en semestres iniciales es un rédito adicional que trae beneficios altamente positivos en semestres posteriores, dado que el concepto de colaboración visto desde la perspectiva de aprendizaje es, en palabras sencillas, lo que permite una complementariedad que a la postre será beneficiosa para la sociedad, que espera a esos futuros y nuevos ingenieros. Las opiniones de los estudiantes evidencian que es tan importante el concepto de colaboración mutua, como la competencia sana que se puede generar entre los grupos y, por momentos, entre sus integrantes. No es menospreciable la forma como ellos mismos destacan la importancia de la interacción, así como la de las responsabilidades que se les asignan.

La adopción de un modelo de clasificación de estudiantes, que en este caso se refiere al modelo 4Q de preferencias de pensamiento, podría refinarse un poco más si se centra la atención en la forma de aproximarse a su cuadrante preferente, pero sin desconocer que el tiempo apremia en un semestre de dieciséis semanas. De allí que la comunicación, el diálogo y la interacción entre docentes y estudiantes (tan necesaria y tan posible en tiempos modernos) sea otro derivado de este tipo de proyectos de investigación, que no solo permite dicha aproximación, sino que además posibilita que se conozca un poco más de los estudiantes (sin desconocer que podrían existir algunos errores naturales en este proceso de investigación).

\section{REFEREnCiAs}

Ausubel, D. (1986). Sicología educativa: un punto de vista cognoscitivo. Ciudad de México: Trillas.

Barrell, J. (2006). Problem based learning: an inquiry approach (2a ed.). New York: Corwin. 
Bonwell, C., y Eison, J. (1991). Active learning: creating excitement in the classroom (ASH-EERIC Higher Education Report n. ${ }^{\circ}$ 1). Washington, D. C.: The George Washington University, School of Education and Human Development

Collazos, C., Guerrero, L., Pino, J., y Ochoa, S. (2002). Evaluating Collaborative Learnng Processes. En J. Haake and J. Pino (Eds.), CRIWG 2002, LNCS 2440, (pp. 203-221). Berlin: Springer.

Dooly, M. (2008). Constructing knowledge together. En M. Dooly (ed.), Telecollaborative Language Learning. A guidebook to moderating intercultural collaboration online (pp. 21-45). Bern: Peter Lang.

Duch, B., Groh, S., y Allen, D. (2001). The power of Problem Based Learning. Delaware, USA: Falmer/KP.

Gillies, R. (2007). Cooperative learnig: integrating theory and practice. Brisbane, Australia: SAGE Publications.

Gómez, M., Gómez, P., y González, P. (2007). El estilo de aprendizaje y la relación con el desempeño académico de los estudiantes. Revista Iberoamericana de Inteligencia Artificial, 11(33), 25-36.

Hall, S., Waitz, I., Brodeur, D., Soderholm, D., y Nasr, R. (2002). Adoption of active learning in a lecture based engineering class. 32nd ASEE/IEEE Frontiers in Education Conference, Session $T 2 A$.

Mabrouk, P. (2007). Cooperative learning and active learning: models from the analytical sciences. ACS Symposium series 970, 34-53.

Medina, J. (2012). Los 12 principios del cerebro. Bogotá, Colombia: Grupo Editorial Norma.

Paz, H. (2009). How to develop metacognition through problem solving in higher education? Revista de Ingeniería e Investigación, 31(1), 75-80.

Prince, M. (2004). Does Active Learning work? Journal Engineering Education, 93(3), 223-231.

Small, G. (2011). El cerebro digital. Madird: Editorial Urano.

Trejos, O. (2013). Significado y Competencias. Pereira, Colombia: Papiro. 